\title{
Variaciones de abundancia y biomasa del zooplancton en un embalse tropical oligo-mesotrófico del norte de Venezuela
}

\author{
Sandra Merayo \& Ernesto J. González \\ Universidad Central de Venezuela, Instituto de Biología Experimental, Laboratorio de Limnología, Apartado 47106, \\ Los Chaguaramos, Caracas 1041, Venezuela; ernesto.gonzalez@ciens.ucv.ve
}

Recibido 22-V-2009. Corregido 24-IX-2009. Aceptado 28-X-2009.

\begin{abstract}
Variations of zooplankton abundance and biomass in a tropical oligo-mesotrophic reservoir in Northern Venezuela. Zooplankton abundance in reservoirs is mainly determined by the speed and content of the water, but relatively little is known regarding tropical reservoirs. We studied the seasonal and spatial distribution of zooplankton abundance and biomass along the longitudinal axis of Clavellinos reservoir, northern Venezuela, from October 2006 to September 2007. Zooplankton was collected from the oxygenated layer using a plankton net. A total of 16 taxa were identified: Copepoda, Cladocera, Rotifera, Ostracoda and Diptera. Thermocyclops decipiens (Copepoda, Cyclopoida) dominated the community, while rotifers were the most diverse, with 10 species. Zooplankton abundance varied from 31 to $101 \mathrm{ind} / \mathrm{L}$ in E1, 36 to $84 \mathrm{ind} / \mathrm{L}$ in E2, and, from 30 to $250 \mathrm{ind} / \mathrm{L}$ in E3. Biomass varied from 97.4 to $1406.3 \mu \mathrm{g} / \mathrm{l}$ in E1, 108.5 to $397.2 \mu \mathrm{g} / \mathrm{l}$ in E2, and from 25.9 to $763.9 \mu \mathrm{g} / \mathrm{l}$ in E3. This zooplankton community seems to respond to environmental variations in the reservoir, rather than to variations in resource availability. Rev. Biol. Trop. 58 (2): 603-619. Epub 2010 June 02.
\end{abstract}

Key words: tropical reservoir, zooplankton, abundance, biomass, Clavellinos, Venezuela.

El embalse es un híbrido de río y lago, en el cual el río embalsado regula y retarda su flujo, se extiende en forma de una capa de agua que alcanza un equilibrio más avanzado, tanto en relación con su entorno físico como con el desarrollo de la vida; la tasa de renovación del agua es más lenta que en un río y más rápida que en un lago (Margalef 1983). La organización vertical del lago y la horizontal del río quedan sustituidas por otra organización intermedia y característica, de la que es un elemento importante la asimetría entre la presa o dique y la cola del embalse (Margalef 1983).

Los embalses son sistemas dinámicos complejos sometidos tanto a las condiciones ambientales como a las actividades antropogénicas, las cuales con frecuencia modifican los gradientes verticales y horizontales de las variables limnológicas tales como la penetración de la luz, temperatura del agua, oxígeno disuelto y concentración de nutrientes (Infante 1988), así como también la dinámica generada por las variaciones a corto plazo en el tiempo de residencia de las aguas, régimen de flujo, la salida y el nivel de las mismas, las interacciones con otros ecosistemas acuáticos y terrestres en la zona de captación (Nogueira 2001).

La distribución y abundancia del zooplancton está influenciada por estas condiciones y, adicionalmente, por las interacciones bióticas como la depredación y la competencia (Vanni 1987, Folt \& Burns 1999). Sin embargo, aún no está claro cómo actúan estos factores bióticos en las variaciones horizontales de la comunidad del zooplancton en los embalses (Urabe 1990, López et al. 2001, Villabona-González et al. 2007).

En cuanto a la distribución de la comunidad del zooplancton en estos ecosistemas, varios estudios (Tundisi et al. 1975, Urabe 
1990, Fabián \& Cruz-Pizarro 1997), destacan que la estructura comunitaria cambia gradualmente desde la región fluvial hasta la presa, debido a la diferencia longitudinal que presentan los embalses. Uno de los modelos que mejor explica la hidrodinámica de la zonificación dentro de los embalses es el propuesto por Thornton (1990), en el cual se presenta un esquema de tres regiones (zonas fluvial, de transición y lacustre) para discernir gradientes longitudinales y que consiste en una reducción gradual de la velocidad de la corriente, lo que provoca un aumento de la tasa de sedimentación del material fino suspendido a lo largo del eje longitudinal del ecosistema, desde el río hacia la presa.

Según Marzolf (1990), este gradiente longitudinal que se establece en los embalses presenta una fuente de recursos particulados -entre los cuales se encuentra la comunidad del fitoplancton- que son responsables, en gran medida y la mayor parte del tiempo, de las variaciones de la densidad poblacional, composición de especies y funcionamiento reproductivo del zooplancton. Según este modelo, la abundancia zooplanctónica de cualquier embalse está determinada por dos factores principales, que son la velocidad de la corriente, donde la mayor abundancia se esperaría en la zona cercana a la presa, y un segundo factor generado por la exportación del material (arcilla, nutrientes, carbono orgánico disuelto y microflora), que haría que la mayor abundancia se concentrara en la zona fluvial del embalse, disminuyendo progresivamente hacia la presa. $\mathrm{Si}$ ambos factores estuvieran actuando simultáneamente, la densidad zooplanctónica se asemejaría a una distribución de frecuencia con asimetría positiva, es decir, una mayor abundancia de organismos en la región de transición del embalse.

En este estudio se documentan por primera vez las variaciones horizontales y temporales de la abundancia y biomasa del zooplancton del embalse Clavellinos (Venezuela), con el objetivo de establecer patrones de distribución espacial durante los períodos de estratificación y mezcla de las aguas.

\section{MATERIALES Y MÉTODOS}

Área de estudio: el embalse Clavellinos se encuentra ubicado en la región noreste de Venezuela sobre el río Clavellinos, entre los $10^{\circ} 19$ $10^{\circ} 23^{\prime} \mathrm{N}, 63^{\circ} 35^{\prime}-63^{\circ} 40^{\prime} \mathrm{W}$ a $296 \mathrm{~m}$.s.n.m. (Fig. 1). Fue construido para el suministro de agua potable a una población de 512366 habitantes, además de suministrar agua para irrigación de unas 6 200ha (Ferraz-Reyes \& Fernández 1988). Tiene un área de 1050 ha, un volumen de $131 \mathrm{hm}^{3}$, una profundidad media de $12.5 \mathrm{~m}$ y un tiempo de residencia teórico de aproximadamente de 106 días.

Métodos y análisis: se recolectaron muestras mensuales durante el período comprendido entre octubre 2006 y septiembre 2007, con el fin de abarcar los períodos climáticos de lluvias (octubre a diciembre 2006 y de junio a septiembre 2007) y sequía (enero a mayo 2007). Para ello, se establecieron dos localidades en los extremos del eje longitudinal del embalse, en la región fluvial del embalse y en la zona cercana a su dique, siguiendo el criterio de Thornton (1990), y una tercera localidad, cercana al punto medio del cuerpo de agua, que pudiera representar la zona de transición del citado modelo:

Estación lacustre (E1): zona lacustre, cercana al dique y al punto de extracción de aguas para suministro. Presentó una profundidad promedio de $28 \mathrm{~m}$ durante el periodo de estudio.

Estación de transición (E2): zona de transición. Presentó una profundidad promedio de $24 \mathrm{~m}$.

Estación fluvial (E3): zona fluvial, ubicada cerca de la desembocadura del río Clavellinos en el embalse. Presentó una profundidad promedio de $4 \mathrm{~m}$. El fondo de esta estación estuvo cubierto por un denso manto de macroalgas (Chara sp.).

Las localidades E1 y E2 fueron consideradas estaciones profundas o limnéticas, mientras que E3 fue la estación somera. 


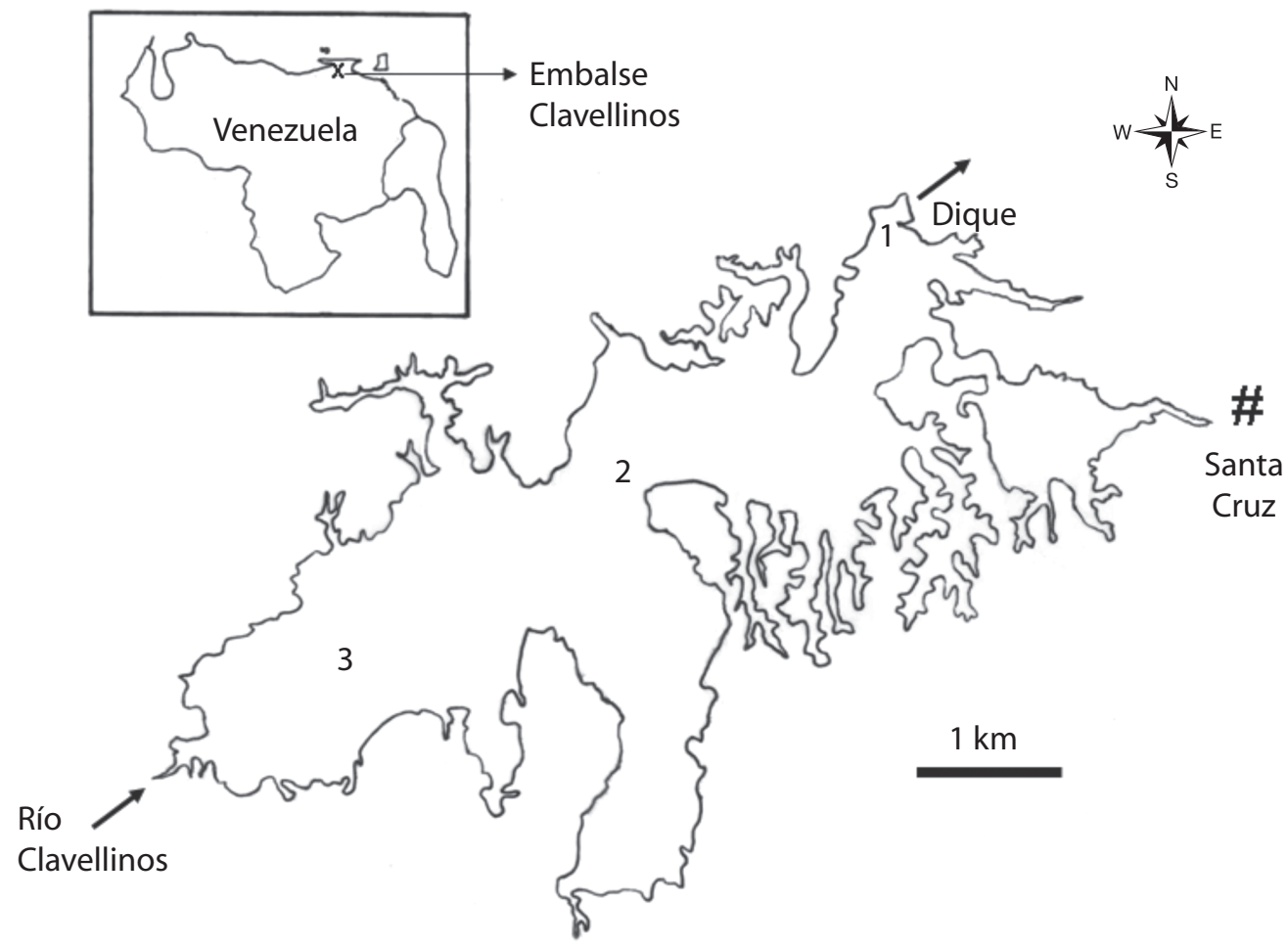

Fig. 1. Estaciones de muestreo en el embalse Clavellinos.

Fig. 1. Sampling sites in Clavellinos reservoir.

Parámetros ambientales: En cada estación se midieron la transparencia del agua mediante un disco de Secchi de $20 \mathrm{~cm}$ de diámetro, la temperatura del agua $\left(0.1^{\circ} \mathrm{C}\right.$ de precisión), oxígeno disuelto $(0.01 \mathrm{mg} / 1$ de precisión $)$ y conductividad ( $1 \mu \mathrm{S} / \mathrm{cm}$ de precisión) con una sonda multiparamétrica YSI, modelo 610-DM y el $\mathrm{pH}$ con un potenciómetro portátil Oakton Testr $1^{\mathrm{TM}}$.

También se tomaron muestras de agua con la ayuda de una botella de captación del tipo van Dorn (51) para los análisis de nitrógeno total $\left(\mathrm{N}_{\mathrm{T}}\right)$ y fósforo total $\left(\mathrm{P}_{\mathrm{T}}\right)$. Las muestras se mantuvieron en frío y en oscuridad hasta su análisis en el laboratorio.

Parámetros biológicos: Las muestras de zooplancton se recolectaron en las tres estaciones de muestreo mediante una red de cierre de $77 \mu \mathrm{m}$ de luz de malla con la que se realizaron arrastres verticales a intervalos de 2 metros (volumen filtrado $=62.8$ litros) en el estrato oxigenado de la columna de agua. Las muestras se preservaron con solución de formalina al 4\% de concentración final, hasta su análisis posterior en el laboratorio (Wetzel \& Likens 2000).

Con la ayuda de la botella de captación, se recolectaron muestras de agua en la zona eufótica del embalse para la estimación de la concentración de clorofila $a$ del fitoplancton. Las muestras se almacenaron en frío y en oscuridad hasta su análisis en el laboratorio.

Análisis de laboratorio: Las concentraciones de $\mathrm{N}_{\mathrm{T}} \mathrm{y}_{\mathrm{T}}$ se determinaron simultáneamente 
mediante la digestión de las muestras con persulfato de potasio en autoclave a $110^{\circ} \mathrm{C}$ durante 30min (Valderrama 1981). Las determinaciones se realizaron con la ayuda de un espectrofotómetro Milton Roy Spectronic 401 a 543nm para el $\mathrm{N}_{\mathrm{T}}$ y a $882 \mathrm{~nm}$ para el $\mathrm{P}_{\mathrm{T}}$.

La concentración de clorofila $a$ se determinó mediante la extracción de pigmentos fotosintéticamente activos con etanol a $75^{\circ} \mathrm{C}$ luego de filtrar $250 \mathrm{ml}$ de muestra a través de filtros de fibra de vidrio Whatman GF/C (Nusch \& Palme 1975). Las determinaciones se realizaron con la ayuda de un espectrofotómetro Milton Roy Spectronic 401 ${ }^{\mathrm{TM}}$.

Las estimaciones de abundancia del zooplancton se realizaron tomando alícuotas de $1 \mathrm{ml}$ de muestras concentradas $(30 \mathrm{ml})$ y analizadas en cámaras de Sedgwick-Rafter y la posterior observación e identificación al microscopio con la ayuda de claves taxonómicas (Wetzel \& Likens 2000). Los conteos se realizaron por duplicado. La biomasa se estimó como peso seco, y se determinó por duplicado mediante el desecado de $0.3 \mathrm{ml}$ de muestra por $20-24 \mathrm{~h}$ a $60^{\circ} \mathrm{C}$ y su posterior pesado en ultramicrobalanza (1 $\mu \mathrm{g}$ de precisión) (Edmondson \& Winberg 1971).

Mediante un ANOVA de una vía, previa comprobación de los supuestos para su aplicación (Sokal \& Rohlf 1979), se determinó si hubo diferencias significativas $(\mathrm{p}<0.05)$ entre las variables fisicoquímicas analizadas. Se aplicó la prueba "a posteriori” de diferencia mínima significativa para determinar cuál o cuáles fueron las localidades homogéneas. Las pruebas se realizaron con el programa STATISTICA ${ }^{\mathrm{TM}}$ para Windows v. 7.0 (StatSoft Inc. 2004).

La inexistencia de normalidad y homogeneidad de varianza determinó la aplicación de la prueba de Kruskal-Wallis (H) (Siegel \& Castellan 1995) para evaluar si la abundancia y biomasa del zooplancton fueron significativamente diferentes entre las estaciones de muestreo. Para los resultados significativos, se realizó la prueba a posteriori de Mann-Whitney (U) (Siegel \& Castellan 1995) con la finalidad de comparar diferencias y similitudes entre estaciones. Para ambas pruebas se planteó un nivel de significación de 95\% y se realizaron con el programa STATISTICA para Windows v. 7.0 (StatSoft Inc. 2004).

A fin de describir las relaciones entre las variables ambientales y la estructura de la comunidad del zooplancton, se aplicó un análisis de redundancia (RDA) centrando la matriz de especies y transformándolos logarítmicamente. Las variables ambientales incorporadas al modelo fueron escogidas a través de una selección de tipo "forward" (Ter Braak \& Smilauer 1998a) y una matriz de especies cuyos taxones fueron considerados constantes y comunes. También se realizó una prueba de Monte Carlo para determinar la significancia estadística $(\mathrm{p}<0.05)$ de la relación entre especies y las variables ambientales estimadas. Estos análisis fueron realizados con el programa CANOCO para Windows v. 4.5 (Ter Braak \& Smilauer 1998b).

\section{RESULTADOS}

Parámetros ambientales: En la región de estudio, según datos suministrados por el Ministerio del Ambiente de Venezuela, la temporada de lluvias se extiende desde junio a diciembre, con un máximo de precipitación de $151.0 \mathrm{~mm}$ en agosto, mientras que la de sequía transcurre entre enero y mayo, con un mínimo de $18.1 \mathrm{~mm}$ de precipitación en marzo.

Los mayores valores de transparencia (Fig. 2) fueron encontrados en E1 con un valor promedio de $2.31 \pm 0.7 \mathrm{~m}$ y una variación entre 1.3 (diciembre 2006 y enero 2007) y $3.2 \mathrm{~m}$ (mayo y julio 2007), seguido por E2 con un promedio de $1.85 \pm 0.6 \mathrm{~m}$ y una variación entre 1.1 (octubre 2006) y $3.1 \mathrm{~m}$ (agosto 2007), mientras que los menores valores fueron registrados en E3 con una media $1.64 \pm 0.3 \mathrm{~m}$ y una fluctuación entre 1.1 (octubre 2006) y $2.3 \mathrm{~m}$ (agosto 2007). Se detectaron diferencias significativas entre las localidades estudiadas $(\mathrm{F}=4.15, \mathrm{p}<0.05)$; sólo se registró que hubo diferencias entre E1 y E3.

El embalse presentó una circulación completa en la época de sequía, en los meses de febrero y marzo, permaneciendo estratificado térmicamente el resto del período de estudio, 
Tiempo (meses)

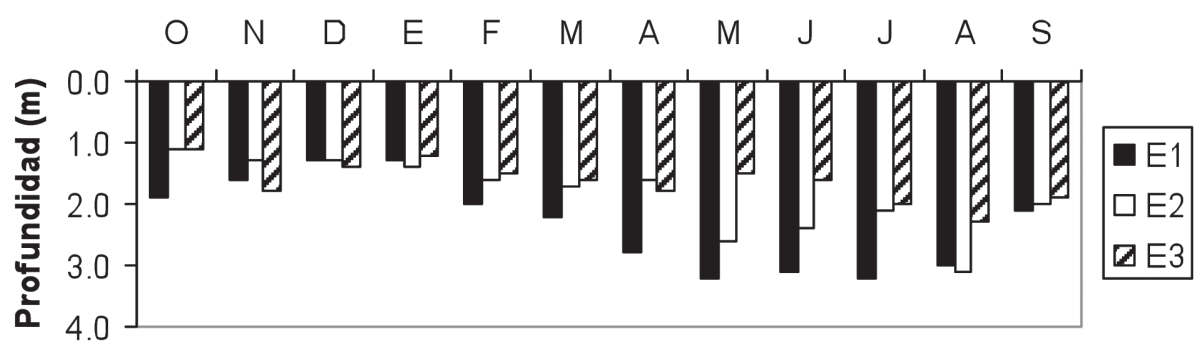

Fig. 2. Variaciones temporales de la transparencia del agua en las tres estaciones de muestreo.

Fig. 2. Temporal variations of water transparency in the three sampling sites.

cuando presentó una diferencia promedio de temperatura entre la superficie y el fondo de $1.6^{\circ} \mathrm{C}$, mientras que para los meses de circulación se registró una disminución de esta diferencia, la cual no superó los $0.4^{\circ} \mathrm{C}$.

El patrón de variación del oxígeno disuelto en E1 (Fig. 3) fue similar al resto de las localidades estudiadas. Los valores superficiales de oxígeno disuelto oscilaron entre 5.9 y $9.5 \mathrm{mg} / 1$ y se presentaron condiciones de hipoxia y anoxia a partir de 15 metros de profundidad en las estaciones limnéticas durante el período de estratificación térmica, acompañado además de un olor a sulfuro de hidrógeno, el

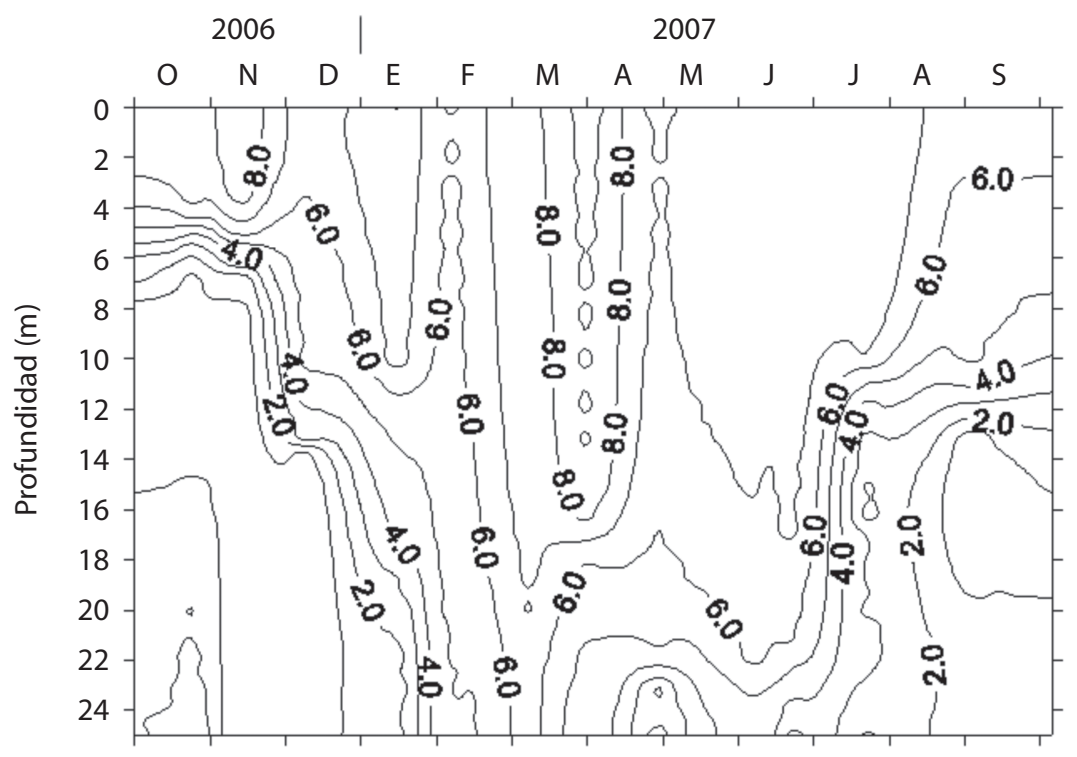

Fig. 3. Isopletas de oxígeno (mg/l) en E1 del embalse Clavellinos.

Fig. 3. Isopleths of oxygen (mg/l) in E1 of Clavellinos reservoir. 
cual es un indicador de que durante este periodo existió una alta tasa de descomposición en los estratos profundos y que su concentración

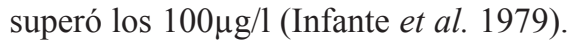

La conductividad promedio registrada durante el período de estudio fue siempre menor a $270 \mu \mathrm{S} / \mathrm{cm}$. El mínimo valor de $172 \mu \mathrm{S} / \mathrm{cm}$ se registró durante la temporada de lluvias.

Las concentraciones de nitrógeno total y fósforo total fueron de relativamente bajas a moderadas (Cuadro 1), mientras que el carácter de las aguas fue ligeramente básico, encontrándose valores máximos de $\mathrm{pH}$ de 8.7 a nivel superficial y 7.3 en estratos profundos (Cuadro 1). Ninguna de estas variables presentó diferencias significativas $(\mathrm{p}<0.05)$ en la zona eufótica de las localidades de estudio (Cuadro 2).
Parámetros biológicos: $\mathrm{Al}$ considerar la biomasa del fitoplancton (estimada como concentración de clorofila $a$ ) (Fig. 4), el mayor valor registrado se presentó en E1, y fue de $28.42 \mu \mathrm{g} / 1$ (enero 2007) y el mínimo de $9.87 \mu \mathrm{g} / 1$ (junio 2007), con un promedio de $15.41 \pm 5.76 \mu \mathrm{g} / \mathrm{l}$. En E2 se registraron valores extremos de $30.49 \mu \mathrm{g} / \mathrm{l}$ (enero 2007) y $8.29 \mu \mathrm{g} / \mathrm{l}$ (junio 2007) con un promedio de $15.25 \pm 7.15 \mu \mathrm{g} / \mathrm{l}$. En E3, el mayor valor fue de $29.90 \mu \mathrm{g} / 1$ (diciembre 2006) y el menor de $6.51 \mu \mathrm{g} / 1$ (julio 2007), con un promedio de $15.00 \pm 7.65 \mu \mathrm{g} / \mathrm{l}$. Se registraron concentraciones mayores durante la época de sequía, pero no se detectaron diferencias significativas $(\mathrm{F}=0.01$, $\mathrm{p}>0.05$ ) entre cada época climática y entre las diferentes localidades de estudio.

CUADRO 1

Intervalos de variación de las concentraciones de nitrógeno total, fósforo total y $\mathrm{pH}$ en el embalse Clavellinos. Entre paréntesis los valores promedios y las desviaciones estándar

TABLE 1

Range of variation of total nitrogen, total phosphorus and $\mathrm{pH}$ in Clavellinos reservoir. In parentheses: mean values and standard deviations

$\begin{array}{lccc} & \text { E1 } & \text { E2 } & \text { E3 } \\ \text { Nitrógeno total }(\boldsymbol{\mu g} / \mathbf{l}) & 111.28-1448.34 & 123.73-533.27 & 123.73-533.4 \\ \text { Superficie } & (411.51 \pm 274.2) & (336.15 \pm 117.5) & (321.28 \pm 123.7) \\ & 123.73-931.08 & 123.73-588.62 & --- \\ \text { Intermedio } & (386.01 \pm 161.9) & (336.57 \pm 125.6) & 123.73-588.82 \\ & 137.48-851.9 & 123.73-766.58 & (363.45 \pm 131.1) \\ \text { Fondo } & (468.24 \pm 166.4) & (390.19 \pm 161.9) & 6.8-21.1 \\ \text { Fósforo total }(\boldsymbol{\mu g} / \mathbf{l}) & & & (12.94 \pm 4.3) \\ \text { Superficie } & 6.8-13.9 & 6.9-81.0 & --- \\ & (9.60 \pm 2.4) & (16.63 \pm 20.5) & 11.3-29.6 \\ \text { Intermedio } & 4.5-264.3 & 6.8-21.4 & (16.73 \pm 6.1) \\ & (19.27 \pm 43.5) & (12.0 \pm 4.5) & 8-8.6 \\ \text { Fondo } & 6.8-608.9 & (29.37 \pm 44.0) & (8.24 \pm 1.3) \\ \text { pH } & (74.67 \pm 165.1) & & ---\end{array}$


CUADRO 2

Análisis de varianza de $N_{T}, P_{T}$ y $\mathrm{pH}$ en las tres estaciones de muestreo

TABLE 2

Analysis of variance for $N_{T} P_{T}$ and $\mathrm{pH}$ in the three sampling sites

$\begin{array}{cccc}\text { Variables } & \mathrm{SS}^{2} & \mathrm{~F} & \text { Nivel significancia } \\ \mathrm{N}_{\mathrm{T}} & 28081.62 & 0.937 & 0.401952 \\ \mathrm{P}_{\mathrm{T}} & 197.379 & 0.701 & 0.503323 \\ \mathrm{pH} & 0.008 & 0.184 & 0.832643\end{array}$

En la comunidad zooplanctónica del embalse se identificaron 16 taxones, 10 rotíferos, 3 cladóceros, 1 copépodo, 1 ostrácodo y 1 díptero (Cuadro 3 ).

La abundancia promedio total del zooplancton disminuyó hacia las zonas limnéticas (E1 y E2) (Fig. 5), encontrándose en E1 un promedio $62 \pm 22 \mathrm{ind} / 1$, con una variación entre $31 \mathrm{ind} / 1$ (octubre 2006) y $101 \mathrm{ind} / 1$ (septiembre 2007). En E2, el promedio fue de $60 \pm 13$ ind/l, con una fluctuación entre 36ind/1 (febrero 2007) y 84ind/l (mayo 2007), mientras que en E3 el promedio fue de $111 \pm 67 \mathrm{ind} / 1$, con
CUADRO 3

Taxones del zooplancton identificados en el embalse Clavellinos durante el período de estudio

TABLE 3

Zooplankton taxa identified in Clavellinos reservoir during the study period

\author{
Copepoda Cyclopoida \\ Thermocyclops decipiens (Kiefer, 1929) \\ Cladocera \\ Ceriodaphnia cornuta (Sars, 1886) \\ Diaphanosoma spinulosum (Herbst, 1975) \\ Moina micrura (Kurz, 1874)

\section{Rotifera} \\ Anuraeopsis sp. \\ Brachionus falcatus (Zacharias, 1898) \\ Hexarthra intermedia (Wiszniewski, 1929) \\ Keratella cochlearis (Gosse, 1851) \\ Keratella tropica (Apstein, 1907) \\ Lecane sp. \\ Lepadella sp. \\ Platyias patulus (Müller, 1786) \\ Polyarthra remata (Skorikov, 1896) \\ Trichocerca sp. \\ Otros \\ Chaoborus sp. \\ Ostracoda
}

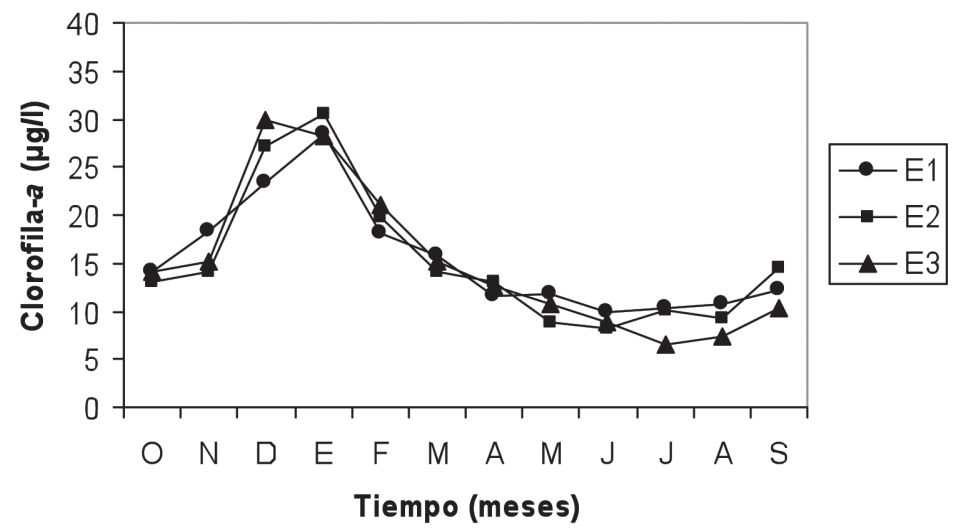

Fig. 4. Variación de la biomasa del fitoplancton (concentración de clorofila $a$ ) en las tres estaciones de muestreo durante el período de estudio.

Fig. 4. Variation of phytoplankton biomass (chlorophyll a concentration) in the three sampling sites during the study period. 


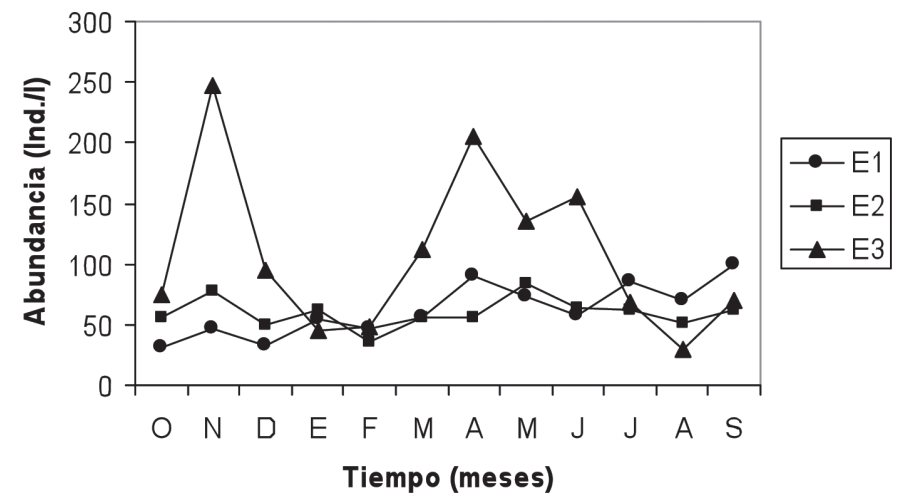

Fig. 5. Variaciones temporales en la abundancia total del zooplancton en las tres estaciones de muestreo.

Fig. 5. Temporal variation in total zooplankton abundance in the three sampling sites.

una variación entre 30ind/1 (agosto 2007) y 250ind/1 (noviembre 2006). Se encontraron diferencias significativas en la abundancia total del zooplancton $(\mathrm{H}=6.70, \mathrm{p}=0.035)$, y $\mathrm{E} 3$ fue diferente a E1 $(\mathrm{U}=37, \mathrm{p}=0.045)$ y $\mathrm{E} 2(\mathrm{U}=30$, $\mathrm{p}=0.014$ ). Por el contrario no se encontraron diferencias significativas entre los períodos climáticos de lluvia y de sequía.

La comunidad zooplanctónica estuvo dominada por el copépodo ciclopoideo Thermocyclops decipiens, que representó el 64.5\% del total. Esta especie se caracterizó por presentar mayor abundancia de nauplios (68\%) y copepoditos $(22 \%)$ que adultos $(10 \%)$. Su abundancia promedio fue de $41 \pm 20 \mathrm{ind} / 1 \mathrm{en} \mathrm{E1}$, $38 \pm 9$ ind/1 en E2 y $67 \pm 43$ ind/1 en E3 (Fig. 6a-c). Sólo se encontraron diferencias significativas en la abundancia de los copepoditos $(\mathrm{H}=7.57$, $\mathrm{p}=0.023)$, ya que $\mathrm{E} 3$ difirió de $\mathrm{E} 1 \quad(\mathrm{U}=28.5$, $\mathrm{p}=0.012)$ y de $\mathrm{E} 2(\mathrm{U}=36, \mathrm{p}=0.038)$.

Los cladóceros se registraron en bajas densidades durante el estudio y representaron el $9.8 \%$ de la abundancia total. Presentaron una abundancia promedio de de $8 \pm 5 \mathrm{ind} / 1$ en E1, seguido por E2 con $7 \pm 4$ ind/l y por último E3 con $6 \pm 6$ ind $/ 1$. Presentaron un incremento a partir de marzo y si bien fueron ligeramente más abundantes en las estaciones E1 y E2, no se encontraron diferencias significativas entre la abundancia registrada en las localidades de estudio (Fig. 6d). De las tres especies encontradas en el embalse, Diaphanosoma spinulosum fue la más abundante, ya que representó el $70.7 \%$ de la abundancia relativa de este grupo, seguida por Ceriodaphnia cornuta (29.1\%) y por último, Moina micrura (0.2\%).

Los rotíferos representaron el $25.7 \%$ del total y presentaron sus mayores abundancias en E3, con un promedio de $34 \pm 32 \mathrm{ind} / 1$, seguido por E2 con $15 \pm 12 \mathrm{ind} / 1$ y por último $13 \pm 11 \mathrm{ind} / 1$ en E1. Se encontraron diferencias estadísticamente significativas en la abundancia total $(\mathrm{H}=6.07$, $\mathrm{p}=0.048$ ), de forma que E3 fue diferente de E1 $(\mathrm{U}=33, \mathrm{p}=0.024)$ (Fig. 6e). No se registraron diferencias entre los períodos climáticos. Keratella cochlearis $(54.0 \%)$ y Brachionus falcatus (46.2\%) dominaron la comunidad en octubre y mayo, respectivamente. Pudo observarse, en general, que pocas especies permanecieron en abundancias relativamente elevadas durante todo el período de estudio.

Al inicio del estudio (noviembre-diciembre), que correspondió con el final del período de estratificación térmica, se encontró una gran abundancia de los rotíferos Keratella cochlearis $(24.4 \%)$, Keratella tropica (7.6\%), Polyarthra remata $(5.3 \%)$ y Platyias patulus $(1.6 \%)$.

Otros grupos (ostrácodos y larvas de Chaoborus sp.) presentaron proporciones extremadamente bajas (0.4\%) (Fig. 7). 

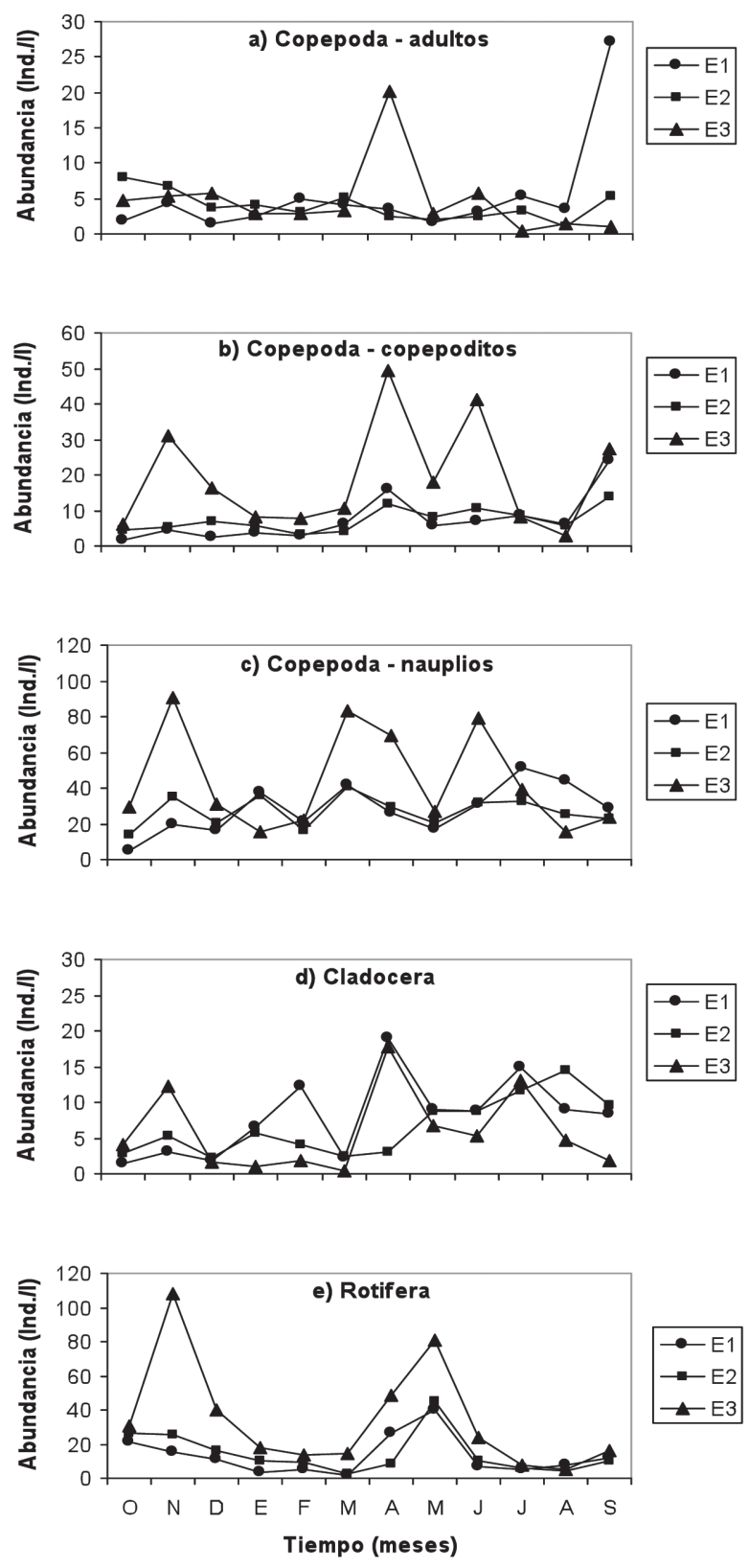

Fig. 6. Variaciones temporales en la abundancia de los grupos del zooplancton en las tres estaciones de muestreo.

Fig. 6. Temporal variations in the abundance of zooplankton groups in the three sampling sites. 
La biomasa promedio del zooplancton en E1 fue de $504.3 \pm 351.8 \mu \mathrm{g} / \mathrm{l}$, con una variación entre $97.4 \mu \mathrm{g} / 1$ (octubre 2006) y $1406.3 \mu \mathrm{g} / 1$ (mayo 2007), mientras que en E2 fue de $371.5 \pm 291.7 \mu \mathrm{g} / \mathrm{l}$, con una fluctuación entre $108.5 \mu \mathrm{g} / 1$ (noviembre 2006) y $397.2 \mu \mathrm{g} / 1$ (septiembre 2007) y, por último, en E3 fue de $312.9 \pm 289.8 \mu \mathrm{g} / 1$, con valores extremos de $25.9 \mu \mathrm{g} / \mathrm{l}$ (febrero 2007) y $763.9 \mu \mathrm{g} / 1$ (agosto 2007) (Fig. 8).

Los mayores valores de biomasa zooplanctónica se encontraron en el período de sequía en E1 y E3, y en el período lluvioso en E2. Sin embargo, no se registraron diferencias estadísticamente significativas entre la biomasa del zooplancton de las localidades estudiadas ni entre los períodos climáticos.
No se registraron correlaciones estadísticamente significativas entre la concentración de clorofila $a$, la abundancia $(\mathrm{r}=-0.56, \mathrm{p}>0.05)$ y biomasa del zooplancton $(r=-0.23, p>0.05)$.

El análisis de redundancia canónica (RDA) (Fig. 9) reveló el patrón de distribución de algunas de las especies que presentaron mayor abundancia y frecuencia de aparición durante el período de estudio. La representación gráfica muestra asociaciones en la distribución de especies en relación a los períodos de estratificación y mezcla.

La varianza acumulada de la abundancia de las especies del zooplancton es explicada por las variables ambientales en un 38.9\%. El análisis con selección "forward" de las variables de predicción indicó que el oxígeno disuelto, transparencia del agua, concentración
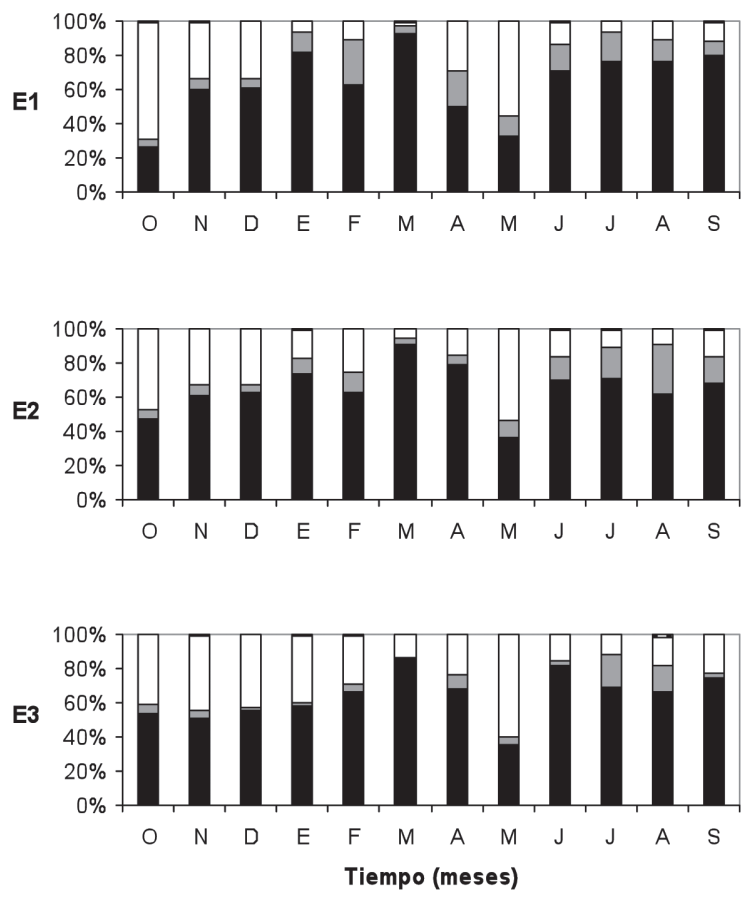

Copepoda $\square$ Cladocera $\square$ Rotifera D Otros

Fig. 7. Proporciones de grupos del zooplancton en el embalse Clavellinos durante el período de estudio en las tres estaciones de muestreo.

Fig. 7. Zooplankton group proportions in Clavellinos reservoir during the study period in the three sampling sites. 


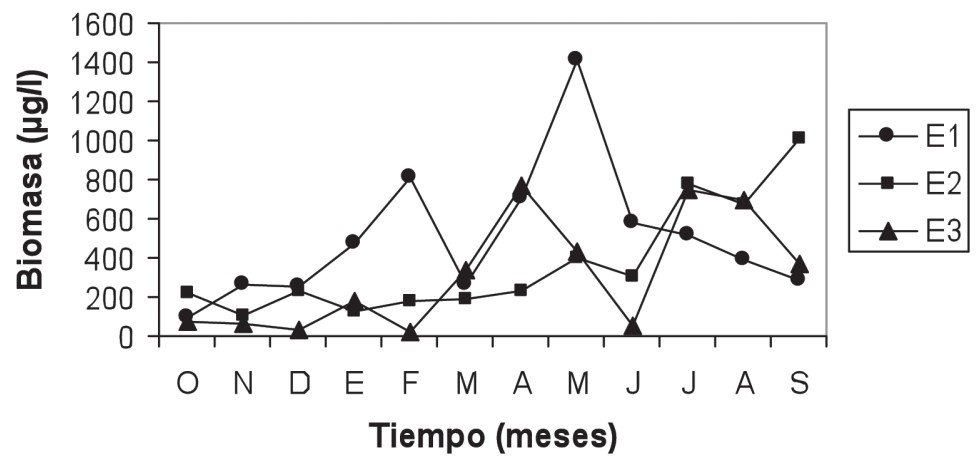

Fig. 8. Variaciones temporales en la biomasa total del zooplancton en las tres estaciones de muestreo.

Fig. 8. Temporal variations of total zooplankton biomass in the three sampling sites.

de clorofila $a$ y temperatura influenciaron significativamente la estructura de la comunidad $(\mathrm{p}<0.05)$.

El test de permutaciones del primer eje de ordenación mostró una relación significativa $(\mathrm{F}=14.4, \mathrm{p}=0.002)$ entre la abundancia de especies y las variables ambientales. La varianza acumulada de la abundancia de las especies fue explicada por las variables ambientales en un $96.3 \%$.

Con la disminución de la temperatura y el incremento de la concentración de oxígeno disuelto hacia los meses de enero, febrero y marzo, que correspondieron al período de mezcla de las aguas, se observó una alta concentración de clorofila $a$, la cual no tuvo relación con el incremento en la abundancia de las especies del zooplancton.

Hacia los meses de transición a la siguiente estratificación térmica (abril-mayo), se observó que el incremento de cladóceros y estadios juveniles de copépodos se relacionaron fuertemente con el incremento de la temperatura y la transparencia del agua.

\section{DISCUSIÓN}

El embalse presentó una circulación completa de sus aguas durante la época de sequía, lo que permitió clasificarlo como cálido monomíctico, según el criterio de Lewis (1983); de baja salinidad, debido a que la conductividad siempre presentó valores menores a los $500 \mu \mathrm{S} /$ $\mathrm{cm}$ (Serruya \& Pollingher 1983) y oligomesotrófico, por valores de $\mathrm{N}_{\mathrm{T}} \mathrm{y}_{\mathrm{T}}$ que pueden considerarse de bajos a moderados (Salas \& Martinó 1991).

La baja riqueza de especies encontrada en el embalse Clavellinos es propia de ambientes con baja concentración de nutrientes. De acuerdo con Matsumura-Tundisi et al. (1990) los ambientes acuáticos eutrofizados ofrecen mayor diversidad de recursos, lo que permite a los organismos que allí habitan una mayor especialización sobre los nichos ecológicos, lo que genera la disminución de la competencia y el incremento de la diversidad.

La abundancia de organismos en el embalse también puede considerarse baja, lo cual resulta propio de ambientes con bajas concentraciones de nutrientes (González et al. 2002). Estos valores se pueden considerar bajos si se les compara con otros embalses de Venezuela estudiados por Infante et al. (1992), como Camatagua, Guanapito y Lagartijo $\left(\mathrm{P}_{\mathrm{T}}\right.$ alrededor de $10 \mu \mathrm{g} / 1$ y clorofila $a$ entre 3 y $20 \mu \mathrm{g} / 1$ ), en los cuales se registraron valores mínimos de $300 \mathrm{ind} / 1$, y en casos extremos como el embalse hipereutrófico Pao-Cachinche $\left(\mathrm{P}_{\mathrm{T}}=462 \mu \mathrm{g} / \mathrm{l}\right.$ y clorofila $a=42 \mu \mathrm{g} / \mathrm{l}$ ), donde se registraron valores que superaron los 1 000ind/1 (González et al. 2002, González et al. 2004), con lo cual se 


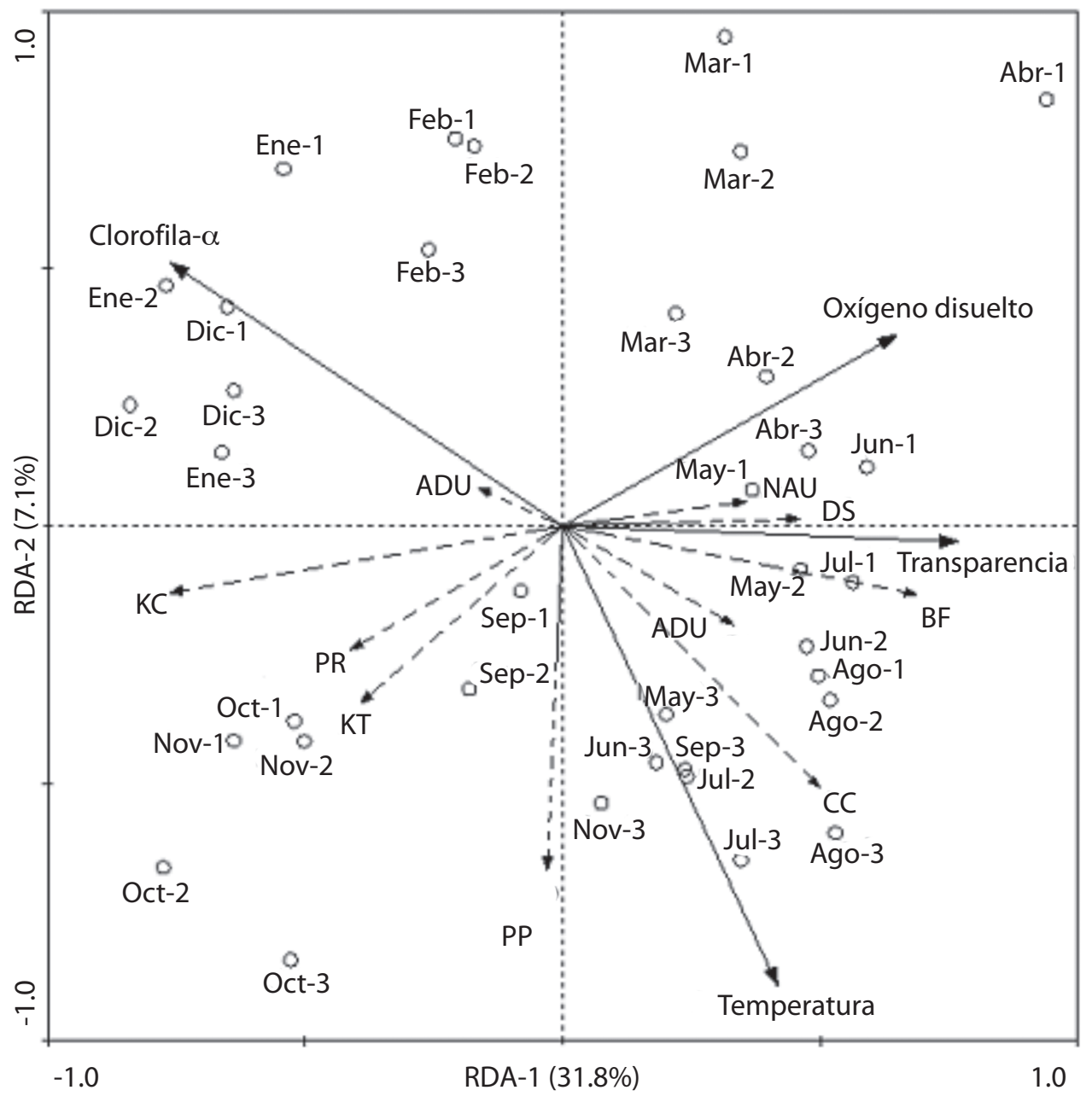

Fig. 9. Análisis de redundancia (RDA). Los círculos son el conjunto de características físicoquímicas, concentraciones de clorofila $a$ y las abundancias de las especies consideradas en el análisis, indicando el mes y la localidad de muestreo correspondiente. Las flechas discontinuas son las especies de zooplancton, las líneas continuas las variables ambientales. $\mathrm{ADU}=$ Copépodos adultos, $\mathrm{COP}=\mathrm{Copepoditos}, \mathrm{NAU}=$ nauplios, $\mathrm{CC}=$ Ceriodaphnia cornuta, $\mathrm{DS}=$ Diaphanosoma spinulosum, $\mathrm{BF}=$ Brachionus falcatus, $\mathrm{KC}=$ Keratella cochlearis, $\mathrm{KT}=$ Keratella tropica, $\mathrm{PR}=$ Polyarthra remata, $\mathrm{PP}=$ Platyias patulus .

Fig. 9. Redundancy Analysis (RDA). Circles represent the overall physicochemical characteristics, chlorophyll $a$ concentrations and abundance of species considered in the analysis, indicating month and location for each sampling. Discontinuous arrows represent zooplankton species, while continuous lines represent environmental variables. $\mathrm{ADU}=\mathrm{Adults}$ copepods, $\mathrm{COP}=$ Copepodits, $\mathrm{NAU}=$ nauplii, $\mathrm{CC}=$ Ceriodaphnia cornuta, $\mathrm{DS}=$ Diaphanosoma spinulosum, $\mathrm{BF}=$ Brachionus falcatus, $\mathrm{KC}=$ Keratella cochlearis, $\mathrm{KT}=$ Keratella tropica, $\mathrm{PR}=$ Polyarthra remata, $\mathrm{PP}=$ Platyias patulus. 
puede inferir que la densidad del zooplancton posee una relación directa con el grado de eutrofización. Observaciones similares fueron registradas por Sendacz et al. (2006) en los embalses brasileños Ponte Nova (oligotrófico) y Guarapiranga (eutrófico), en los que las abundancias promedio fueron de $69 \mathrm{ind} / 1$ y $678 \mathrm{ind} / 1$, respectivamente.

Contrario a lo propuesto por varios autores que exponen que los rotíferos dominan las comunidades del zooplancton en lagos y embalses tropicales y subtropicales (Sendacz et al. 2006), el copépodo Thermocyclops decipiens dominó durante casi todo el período de estudio. Al respecto, Santos-Wisniewski \& Rocha (2007) señalan que esto se puede deber al largo tiempo de residencia de las aguas, lo cual favorece a las especies con un tiempo de desarrollo relativamente largo como el de los copépodos. Thermocyclops decipiens es característico del zooplancton dulceacuícola tropical y ha sido considerado una especie asociada con ambientes eutróficos o perturbados (Sampaio et al. 2002). En un estudio realizado por estos autores en siete embalses del río Paranapanema en Brasil, se encontró que en la mayoría de ellos la especie dominante fue T. decipiens. La misma tendencia fue encontrada por López et al. (2001) en los embalses venezolanos Guri, El Cují, El Andino, Camatagua, entre otros, y señalaron que, por lo general, los copépodos constituyen el grupo más abundante en los embalses venezolanos durante la mayor parte del año.

La comunidad de rotíferos presentó la mayor riqueza de especies, y además fue la más sensible a los cambios ambientales que se produjeron durante el estudio, ya que fueron pocas las especies que permanecieron en abundancias relativamente elevadas. Usualmente la comunidad de rotíferos no exhibe un patrón regular a los cambios estacionales en los lagos tropicales (Matsumura-Tundisi et al. 1990) y geográficamente representan el grupo más extendido por tener estrategias como la reproducción partenogenética, ciclo de vida corto, tamaño pequeño, entre otras, que les permiten una distribución amplia (Sendacz 1993), y la posibilidad de colonizar rápidamente ambientes perturbados. Los géneros Brachionus y Keratella suelen ser típicamente dominantes en los cuerpos de agua tropicales, y en Venezuela las especies más representativas son Keratella americana, Keratella tropica y Brachionus falcatus, entre otras (López et al. 2001). Tal es el caso del embalse Clavellinos, donde $K$. cochlearis y un representante de la familia Brachionidae $(B$. falcatus) dominaron en los meses octubre y mayo, respectivamente.

Por su parte, los cladóceros también presentan una amplia distribución en ambientes tropicales, sin embargo, a veces ésta se ve restringida a pequeñas áreas debido a los requerimientos ecológicos y fisiológicos que presentan algunas especies (Sendacz 1993). En el embalse Clavellinos se encontraron $D$. spinulosum, C. cornuta y M. micrura. Particularmente, en los embalses de Venezuela la asociación de estas especies es la más común entre los cladóceros (López et al. 2001). Esta misma composición comunitaria de cladóceros también fue encontrada en el embalse oligotrófico brasileño Jucazinho (Melo Júnior et al. 2007).

Varios autores exponen que el zooplancton de sistemas oligotróficos responde de manera directa a las variaciones del fitoplancton (Gliwicz 1969, Infante 1988). Esto ha sido encontrado en los embalses oligotróficos venezolanos Taguaza (González et al. 2002) y Agua Fría (González 2002), en los que se registraron correlaciones positivas y significativas entre la concentración de clorofila $a$ y la biomasa del zooplancton. Sin embargo, este no fue el caso del embalse Clavellinos, coincidiendo con los resultados de Gavidia (2003) en el embalse Tierra Blanca. Gavidia (2003) expresa que, en este caso, la biomasa del zooplancton pudo haber sido afectada por otro factor, más que por la cantidad de alimento, posiblemente por la depredación.

Al respecto, Brooks \& Dodson (1965) proponen que la estructura de tallas del zooplancton puede ser afectada por la depredación selectiva de algunos peces. En el embalse Clavellinos, aunque no se estudió la composición de la comunidad de peces y su efecto en la 
comunidad del zooplancton, se pudo observar la presencia de la especie Caquetaia kraussii; en la actualidad, esta especie se encuentra presente en varios ríos y embalses venezolanos. La depredación que pudiera ejercer esta especie sobre el zooplancton podría explicar la baja densidad de cladóceros y copépodos adultos en el embalse, principalmente en E3. Una situación similar fue registrada por Ortaz et al. (2006), en el embalse hipereutrófico Pao-Cachinche (Venezuela), donde encontraron que el orden de importancia de las presas planctónicas en la dieta de C. kraussii fue como sigue: Cladóceros $>$ Chaoborus sp.>copépodos>rotíferos. Así, en Clavellinos, la remoción de los organismos de tallas mayores por depredación pudiera haber generado la independencia entre la biomasa de fitoplancton y la del zooplancton, ya que al ser eliminados los consumidores de tallas mayores (cladóceros y copépodos), se puede ejercer una menor presión sobre la comunidad del fitoplancton.

Los mayores valores de biomasa del zooplancton coincidieron con los picos de abundancia del zooplancton, registrados durante el período de transición de la mezcla completa de las aguas a la estratificación térmica. La biomasa total del zooplancton de las localidades del embalse Clavellinos fueron superiores a las registradas en los embalses oligotróficos Ponte Nova (Brasil, 14.1 $\mu \mathrm{g} / \mathrm{l})$ (Sendacz et al. 2006) y Taguaza (Venezuela, 28.7 $\mu \mathrm{g} / \mathrm{l}$ ) (González et al. 2002), similares al máximo registrado en el embalse eutrófico brasileño de Guarapiranga (356.8 $\mu \mathrm{g} / 1$ durante la época de sequía) (Sendacz et al. 2006), e inferiores a los registrados por Carrillo (2001) en el embalse eutrófico La Mariposa (Venezuela, promedios entre 273.5 y $1056.2 \mu \mathrm{g} / 1)$.

En cuanto a la distribución, Ricklefs (1990) sugiere que los arreglos comunitarios no son consecuencia del azar, sino que existen mecanismos que actúan sobre los ecosistemas, produciendo patrones u ordenamientos particulares. Según Ford (1990), la heterogeneidad horizontal de los parámetros limnológicos en los embalses se debe a los procesos de transporte, principalmente a fuerzas advectivas, las cuales deben ser consideradas como una condición general presente en cualquier embalse, que generan además condiciones de hábitat diferentes para el establecimiento de animales $\mathrm{y}$ vegetales que mejor se adapten a ellos.

El factor de la exportación del material (Marzolf 1990) pareció ser el dominante $y$, por lo tanto, el más adecuado para explicar los valores de abundancia del zooplancton registrados en el embalse. Sin embargo, pudo notarse que los cladóceros presentaron una mayor abundancia hacia la región lacustre, lo cual pudo deberse a la gran cantidad de sólidos totales presente en la zona fluvial (Machado 2008) que redujo la abundancia de especies del género Ceriodaphnia, el cual posee una baja tolerancia a estas condiciones (Lougheed \& Chow-Fraser 1998), además de utilizar la luz para orientarse, por lo que exhiben preferencias por los ambientes con mejor calidad lumínica, como sería el caso de las localidades profundas (limnéticas). Esta preferencia por ambientes con gran penetración de luz permite una mayor depredación por parte de los peces zooplanctófagos (Brönmark \& Hansson 2001). Sin embargo, estos filtradores poseen un amplio intervalo en cuanto al tamaño de partícula de las cuales se alimentan, que los hace menos vulnerables a las grandes fluctuaciones de abundancia de un tamaño de partícula en específico, y probablemente debido a este mecanismo de alimentación, se pudiera explicar la permanencia de estos organismos durante todo el período de estudio.

Por el contrario los rotíferos fueron más abundantes hacia la región fluvial, posiblemente debido a que los altos niveles de sólidos inorgánicos suspendidos -hasta 265mg/l- (Machado 2008) actúan de cierta manera en contra de la selección de grandes filtradores, permitiendo a las especies de zooplancton de talla pequeña ser dominantes bajo estas condiciones (Lougheed \& Chow-Fraser 1998). Además, la mayoría de los rotíferos son "suspensívoros" y se alimentan básicamente de partículas detríticas muy pequeñas, las cuales no son utilizados por organismos de talla grande como los copépodos y cladóceros, lo que confiere ventajas a los 
rotíferos, ya que son capaces de alimentarse de estas partículas, aun cuando el fitoplancton, la principal fuente de recursos para el zooplancton, se encuentre en bajas concentraciones.

A diferencia de las otras estaciones, en E3 la presencia de un denso lecho de Chara sp. pudo haber conferido sitios de refugio a los organismos (Jeppesen et al. 1997, Jeppesen et al. 1999), lo que posiblemente permitió un menor riesgo de depredación por peces, y que se generaran las altas abundancias y dominancia de los estadios juveniles de copépodos y de rotíferos que se registraron en esta estación, sobre todo al considerar que en los sistemas lénticos tropicales los peces ejercen un control casi permanente del zooplancton, ya que su extenso período reproductivo favorece la presencia de estadios juveniles predominantemente zooplanctófagos a lo largo del año (Zaret 1980).

Como se pudo constatar, las variaciones en la composición, abundancia y biomasa del zooplancton coincidieron con la modificación de condiciones físicas y químicas de las aguas durante el período de sequía y de lluvias, más que por las variaciones de la concentración de clorofila $a$. En síntesis, se observó que la comunidad tuvo una dinámica que se relacionó fuertemente con las condiciones ambientales, regidas principalmente por los períodos de estratificación y mezcla de las aguas más que por la concentración de clorofila $a$.

Los estudios sobre la estructura y funcionamiento de la comunidad del zooplancton dan la oportunidad de investigar los patrones de respuesta a las variaciones cíclicas y los eventos de perturbación de los embalses (Nogueira 2001). En este último aspecto, MatsumuraTundisi (1997) expone que la dinámica poblacional de los diversos grupos de zooplancton constituye un eslabón relevante en el manejo de los embalses, ya que la composición, abundancia y distribución espacial de las comunidades zooplanctónicas están fuertemente relacionadas con el estado trófico y el grado de interacciones biológicas que ocurren en estos ecosistemas, y que además la prevalencia de ciertas especies puede, de esta forma, ser un indicador del estado trófico del ecosistema.
Finalmente, se debe expresar que es importante reconocer que las zonas de un embalse, según el modelo de Thornton (1990), usualmente son dinámicas y se expanden y contraen en respuesta a los eventos de escorrentía en las cuencas, las características de la densidad del flujo y de la operación del embalse, todo lo cual afecta el desarrollo de las comunidades planctónicas que habitan en este tipo de cuerpo de agua (Kimmel et al. 1990).

\section{AGRADECIMIENTOS}

Los autores desean dejar constancia de su agradecimiento a las siguientes instituciones y personas por el apoyo logístico en los trabajos de campo y de laboratorio: HIDROVEN, HIDROCARIBE, Cooperativa "Rafael Vegas León", Laboratorio de Nutrición Mineral de Plantas Silvestres, Coordinación de Investigación de la Facultad de Ciencias - Universidad Central de Venezuela, Pasquale Molinaro, María Leny Matos, Jesús Chucho, Oscana Túpano, Daniel Machado, Lorena Mibelli, Ricardo Martín, Carlos Peñaherrera, Mario Ortaz y Mario Palacios.

\section{RESUMEN}

La abundancia del zooplancton en los embalses está determinada principalmente por la velocidad y el contenido del agua, pero se sabe relativamente poco sobre los embalses tropicales. Se estudió la distribución temporal y espacial de la abundancia y la biomasa del zooplancton en el eje longitudinal del embalse de Clavellinos, en el norte de Venezuela, entre octubre 2006 y septiembre 2007. El zooplancton fue recolectado en el estrato oxigenado con una red de plancton. Un total de 16 taxones fueron identificados: Copepoda, Cladocera, Rotifera, Ostracoda y Diptera. Thermocyclops decipiens (Copepoda, Cyclopoida) dominó la comunidad, mientras que los rotíferos fueron los más diversos, con 10 especies. La abundancia del zooplancton varió de 31 a $101 \mathrm{ind} / 1$ en E1, 36 a 84ind/1 en E2, y, de 30 a 250ind/L en el E3. La biomasa varió de 97,4 a $1406.3 \mu \mathrm{g} / 1$ en E1, 108,5 a $397.2 \mu \mathrm{g} / 1$ en E2, y de 25,9 a $763.9 \mu \mathrm{g} / 1$ en el E3. Esta comunidad de zooplancton pareció responder a las variaciones ambientales en el embalse, más que a las variaciones en la disponibilidad de recursos.

Palabras claves: embalse tropical, zooplancton, abundancia, biomasa, Clavellinos, Venezuela. 


\section{REFERENCIAS}

Brönmark, C. \& L.A. Hansson. 2001. The biology of lakes and ponds. Oxford University, Oxford, England.

Brooks, J.L. \& S. Dodson. 1965. Predation, body size, and composition of plankton. Science 150: 28-35.

Carrillo, V. 2001. Distribución espacial del zooplancton en el embalse La Mariposa (Distrito Capital) de julio a diciembre de 2000. Tesis de Licenciatura, Universidad Central de Venezuela, Caracas, Venezuela.

Edmondson, W.T. \& G.G. Winberg. 1971. A manual on methods for the assessment of secondary productivity in fresh waters. IBP Handbook $\mathrm{N}^{\circ}$ 17. Blackwell, Oxford, England.

Fabián, D. \& L. Cruz-Pizarro. 1997. Variaciones espaciales y temporales del zooplancton en un lago monomíctico eutrófico (Lago Ton-Ton, Uruguay). Limnetica 13: 55-68.

Ferraz-Reyes, E. \& E. Fernández. 1988. Ciclo del fitoplancton y bacterioplancton en el embalse de Clavellinos, estado Sucre, Venezuela. Bol. Inst. Oceanogr. Univ. Oriente 27: 89-104.

Folt, C. \& C.W. Burns. 1999. Biological drivers of zooplankton patchiness. Trends Ecol. Evol. 14: 300-305.

Ford, D.E. 1990. Reservoir transport processes, p. 15-41. In K.W. Thornton, B.L. Kimmel \& F.E. Payne (eds.). Reservoir Limnology: Ecological perspectives. Wiley, Nueva York, EEUU.

Gavidia, J. 2003. Variación en la abundancia y biomasa del zooplancton en dos localidades del embalse Tierra Blanca, Estado Guárico. Tesis de Licenciatura, Universidad Central de Venezuela, Caracas, Venezuela.

Gliwicz, Z.M. 1969. Studies on the feeding of pelagic zooplankton in lakes with varying trophy. Ekol. Pol. 17: 663-708.

González, E.J. 2002. Caracterización limnológica del embalse Agua Fría (Parque Nacional Macarao, Edo. Miranda). Informe técnico presentado al FONACIT. Caracas, Venezuela.

González, E.J., M. Ortaz, M.L. Matos, J. Mendoza, C. Peñaherrera \& V. Carrillo. 2002. Zooplancton de dos embalses neotropicales con distintos estados tróficos. Interciencia 27: 551-558.

González, E.J., M. Ortaz, C. Peñaherrera \& M.L. Matos. 2004. Fitoplancton de un embalse tropical hipereutrófico (Pao-Cachinche, Venezuela): Abundancia, biomasa y producción primaria. Interciencia 29: 548-555.
Infante, A. 1988. El plancton de aguas continentales. Secretaría General de la Organización de los Estados Americanos. Programa Regional de Desarrollo Científico y Tecnológico. Monografía $\mathrm{N}^{\mathrm{o}} 33$. Washington D.C., EEUU.

Infante, A., O. Infante, M. Márquez, W. Lewis \& F. Weibezahn. 1979. Conditions leading to mass mortality of fish and zooplankton in Lake Valencia, Venezuela. Acta Cient. Venez. 30: 67-73.

Infante, A., O. Infante \& T. Vegas. 1992. Caracterización limnológica de los embalses Camatagua, Guanapito y Lagartijo, Venezuela. Proyecto Multinacional del Medio Ambiente y Recursos Naturales. Organización de los Estados Americanos y Universidad Central de Venezuela, Caracas, Venezuela.

Jeppesen, E., M. Sondergaard \& K. Christofferson. 1997. The structuring role of submerged macrophytes. Nueva York, EEUU.

Jeppesen, E., J.P Jensen, M. Sondergaard, T. Lauridsen, L.J. Pedersen \& L. Jensen. 1999. Top-down control in freshwater lakes: role of nutrient state, submerged macrophytes and water depth. Hydrobiologia 343: 151-164.

Kimmel, B.L., O.T. Lind \& L.J. Paulson. 1990. Reservoir primary production, p. 133-193. In K.W. Thornton, B.L. Kimmel \& F.E Payne (eds.). Reservoir Limnology: Ecological perspectives. Wiley, Nueva York, EEUU.

Lewis, W.M. Jr. 1983. Revised classification of lakes based on mixing. Can. J. Fish. Aq. Sci. 40: 1779-1787.

López, C., M. Villalobos \& E. González. 2001. Estudio sobre el zooplancton de los embalses de Venezuela: Estado actual y recomendaciones para futuras investigaciones. Ciencia 9: 217-234.

Lougheed, V \& P. Chow-Fraser. 1998. Factors that regulate the zooplankton community structure of a turbid, hypereutrophic Great Lakes wetland. Can. J. Fish. Aq. Sci. 55: 150-161.

Machado, D. 2008. Caracterización fisicoquímica de las aguas del embalse Clavellinos (Estado Sucre) durante el período octubre 2006 - junio 2007. Tesis de Licenciatura, Universidad de Los Andes, Mérida, Venezuela.

Marzolf, G.R. 1990. Reservoirs as environments for zooplancton, p. 195-208. In K.W. Thornton, B.L. Kimmel \& F.E Payne (eds.). Reservoir Limnology: Ecological perspectives. Wiley, Nueva York, EEUU.

Matsumura-Tundisi, T. 1997. Composition and vertical distribution of zooplankton in Lake Dom Helvécio - MG, Brazil, p. 309-326. In J.G. Tundisi \& Y. Saijo 
(eds.). Limnological Studies on the Rio Doce Valley Lakes, Brazil. Academia Brasileira de Ciencias, Rio de Janeiro, Brasil.

Matsumura-Tundisi T., S.N. Leitão, L.S. Aguena \& J. Miyahara. 1990. Eutrofização da represa de Barra Bonita: Estrutura e organização da comunidade de Rotifera. Rev. Brasil. Biol. 50: 923-935.

Margalef, R. 1983. Limnología. Omega, Barcelona, España.

Melo Júnior, M., V.L.S. Almeida, M.N. Paranaguá \& A.N. Moura. 2007. Crustáceos planctônicos de um reservatório oligotrófico do Nordeste do Brasil. Planktonic crustaceans of an oligotrophic reservoir in Northeastern Brazil. Zoociências 9: 19-30.

Nogueira, M.G. 2001. Zooplankton composition, dominance and abundance as indicators of environmental compartmentalization in Jurumirim reservoir (Paranapanema River), São Paulo, Brazil. Hydrobiologia 455: 1-18.

Nusch, E.A. \& G. Palme. 1975. Biologische Methoden Fur der Praxis der Gewasseruntersuchung, Bestimmung des Chlorophyll- $a$ und Phaeopigmentgehaltes in Oberflachenwasser. GWF-Wasser/Abwasser 116: 562-565.

Ortaz, M., E.J. González \& C. Peñaherrera. 2006. Depredación de peces sobre el zooplancton en tres embalses neotropicales con distintos estados tróficos. Interciencia $31: 1-8$.

Ricklefs, R. 1990. Ecology. Freeman, Nueva York, EEUU.

Salas, H.J. \& P. Martinó. 1991. A simplified phosphorus trophic state model for warm-water tropical lakes. Water Res. 25: 341-350.

Sampaio, E.V., O. Rocha, T. Matsumura-Tundisi \& J.G. Tundisi. 2002. Composition and abundance of zooplankton in the limnetic zone of seven reservoirs of the Paranapanema River. Brazil. Braz. J. Biol. 62: 525-545.

Santos-Winieswski, M.J. \& O. Rocha. 2007. Spatial distribution and secondary production of Copepoda in a tropical reservoir. Braz. J. Biol. 67: 223-233.

Sendacz, S. 1993. Distribuição geográfica de algúns organismos zooplanctônicos na América do Sul. Acta Limnol. Brasil 6: 31-41.

Sendacz, S., S. Caleffi \& J. Santos-Soares. 2006. Zooplankton biomass of reservoirs in different trophic conditions in the state of Sao Paulo, Brazil. Braz. J. Biol. 66: 337-350.
Serruya, C. \& U. Pollingher. 1983. Lakes of the warm belt. Cambridge, Cambridge, England.

Siegel S. \& N.J. Castellan. 1995. Estadística no paramétrica aplicada a la conducta. Trillas, México, México.

Sokal, R.R. \& F.J. Rohlf. 1979. Biometría. Blume, Madrid, España.

Ter Braak, C.J.F. \& P. Smilauer. 1998a. CANOCO reference manual and user guide to Canoco for Windows. Centre for Biometry, Wageningen, Netherlands.

Ter Braak, C.J.F. \& P. Smilauer. 1998b. CANOCO for Windows. v. 4.5. Computer program. Centre for Biometry, Wageningen, Netherlands.

Thornton, K.W. 1990. Perspective on reservoir limnology, p. 1-13. In K.W. Thornton, B.L. Kimmel \& F.E Payne (eds.). Reservoir Limnology: Ecological perspectives. Wiley, Nueva York, EEUU.

Tundisi, T.M., J.G. Tundisi \& C.E. Matheus. 1975. Plankton studies in a lacustrine environment II. Spatial distribution of the zooplankton. Ciênc. Cult. 27: 269-271.

Urabe, J. 1990. Stable horizontal variation in the zooplankton community structure of a reservoir maintained by predation and competition. Limnol. Oceanogr. 35: 1703-1717.

Valderrama, J.C. 1981. The simultaneous analysis of total nitrogen and total phosphorus in natural waters. Mar. Chem. 10: 109-122.

Vanni, M.J. 1987. Indirect effect of predators on agestructured prey populations: Planktivorous fish and zooplankton, p. 149-160. In W.C. Kerfoot \& A. Sih (eds.). Predation: Direct and indirect impacts on aquatic communities, Nueva Inglaterra, EEUU.

Villabona-González, S., R.A. Gavilán-Díaz \& A.L. Estrada-Posada. 2007. Cambios nictemerales en la distribución vertical de algunos microcrustáceos en un lago artificial del Neotrópico (Colombia). Limnetica 26: $75-88$.

Wetzel, R. \& G.E. Likens. 2000. Limnological analyses. Springer-Verlag, Nueva York, EEUU.

Zaret, T.M. 1980. Predation and freshwater communities. Yale, New Haven, EEUU.

\section{REFERENCIAS DE INTERNET}

StatSoft Inc. 2004. STATISTICA for Windows v. 7. Tulsa, OK, USA (Consultado: 13 marzo 2009, http://www. statsoft.com). 
